\title{
Ethical considerations for allocation of scarce resources and alterations in surgical care during a pandemic
}

\author{
Arthur Rawlings $^{1}$ (I) $\cdot$ Lea Brandt ${ }^{2} \cdot$ Alberto Ferreres $^{3,4} \cdot$ Horacio Asbun $^{5} \cdot$ Phillip Shadduck $^{6}$
}

Received: 17 April 2020 / Accepted: 4 May 2020 / Published online: 12 May 2020

(c) Springer Science+Business Media, LLC, part of Springer Nature 2020

\begin{abstract}
The COVID-19 pandemic caused by SARS-CoV-2 is unprecedented in modern history. Its effects on social behavior and health care delivery have been dramatic. The resultant burden of disease and critical illness has outpaced the diagnostic, therapeutic, and health care professional resources of many clinics and hospitals. It continues to do so globally. The allocation of hospital beds and ventilators, personal protective equipment, investigational therapeutics, and other scarce resources has required difficult decisions. Clinical and surgical practices which are standard in normal times may not be standard or safe during the COVID-19 crisis. How can we best adapt as physicians and surgeons? What foundational ethical principles and systems of principle application can help guide our decision-making? Fortunately, a large body of work in medical ethics addresses these questions. Unfortunately, many surgeons and other health care professionals are probably not as familiar with these concepts. This brief communication is intended to provide a concise explanation of ethical considerations which readers may find helpful when addressing allocation of scarce resources and alterations in surgical care brought on by the current pandemic.
\end{abstract}

Keywords Medical ethics $\cdot$ Resource allocation $\cdot$ Clinical guidelines $\cdot$ Pandemic $\cdot$ COVID-19

\section{Preamble}

The allocation of limited resources during a global health care emergency is not normal practice for most physicians, surgeons, and SAGES/EAES members. Those who have

Arthur Rawlings

rawlingsa@health.missouri.edu

1 SAGES Ethics Committee, Adjunct Faculty Center for Health Ethics, Department of Surgery, University of Missouri, One Hospital Drive, Columbia 65212, MO, USA

2 Director Center for Health Ethics, University of Missouri, Columbia, MO, USA

3 Department of General Surgery, University of Buenos Aires, Dr Carlos A. Bocalandro Hospital, Buenos Aires, Argentina

4 Department of Surgery, Center for Surgical Ethics, University of Washington, Seattle, WA, USA

5 Baptist Health Care, Mayo Clinic Florida, Jacksonville, FL, USA

6 SAGES Ethics Committee, Division Chief General Surgery, TOA Surgical Specialists, Duke University, Durham, NC, USA been involved in or studied military medicine recognize that this is analogous to triage during combat medicine, with nuances unique to the specific pandemic characteristics. This article is intended to provide an ethical foundation and background to help us and our health care communities through this remarkable time. It will not provide definitive answers to every particular situation. Decisions of resource allocation and surgical care during pandemics are best made when they are based on a sound ethical framework developed from previous health care emergencies, the most updated, yet evolving, medical and epidemiological information on the current emergency, and local context. One needs to take in consideration that many decisions will be location-specific, time-specific, and evolving as new information is available. Whenever possible, the burden of particularly difficult resource allocation and clinical decisions should be lifted from the front-line care providers and placed upon clinical triage teams comprised of those with a solid understanding of ethical principles, a reputation for clinical expertise and sound reasoning, and an ability to communicate with transparency and clarity. 


\section{The four fundamental ethical principles}

Beneficence, justice, autonomy, and non-maleficence are generally accepted as the four fundamental ethical principles that guide health care delivery. Beneficence is acting in the best interest of others, promoting the well-being of others. Justice is treating people fairly, impartially, and equitably. Autonomy is self-determination, making one's own choices. Non-maleficence is doing no harm, or acting to avoid harm. These four principles are foundational to the patient-physician relationship and to the delivery of surgical care. Under normal circumstances, even if not expert at articulating them, we honor these principles instinctively, almost without thinking about them. They are intuitive to our daily work.

During pandemics and other disasters, however, these principles still exist, but the application of them may need to change. At times, physicians and other health care providers must adapt to the altered and difficult circumstances in which we find our patients, ourselves, and our society. Altered circumstances may require alterations in clinical and surgical care, no longer focusing solely on the well-being of individual patients, but rather focusing also on the well-being of many patients and the larger affected society. In a pandemic, where needs may outstrip resources, the benefit to the whole population takes precedence over the benefit to any one particular patient. This is different than normal. The use of resources for one patient may mean the denial of resources for other patients. A patient's request for resources for care is still to be respected; a lack of fulfillment of that demand does not deny the patient's autonomy, it only acknowledges that at times not all requests for certain interventions can be met. During a pandemic, fair and impartial distribution of critical care is ruled by values that are not normally considered in an adequately resourced environment. Determining the best, most just distribution of limited resources and providing altered but still ethical patient care are the primary foci of this discussion.

\section{Fundamental values to apply during a pandemic}

The foundational ethical principles still apply during a pandemic. There are, however, specific values that help in the decision-making process for the allocation of scare resources. At times, the values will be competing, even potentially mutually exclusive. It will be impossible to fulfill them all simultaneously. That dilemma is enormously stressful to surgeons and other health care professionals who want to help everyone as they normally do. How then can we proceed ethically? We must develop and apply methods or systems of reasoning, accepting that each of them has trade-offs. Recognized values applied in a resource allocation decision process include "maximizing benefits," "most lives saved," "most life-years gained," "equal treatment," "lottery system," "first-come, firstserved," and "prioritize the worst off." Each is explained briefly here.

\section{Maximizing benefits}

This value of maximizing benefits is usually applied as saving either the most lives possible or the most life-years possible. Each of these two approaches has its proper contribution to the decision-making process during a pandemic, even though each is insufficient by itself.

The "most lives saved" strategy recognizes that each individual life is valuable. With all other things being equal, saving five lives instead of saving one life needs little moral justification. But all things are not equal during a pandemic. For example, is saving the life of three older individuals who should each live another 5 years equal to saving the life of one younger person who should live another 60 years? What if those three elderly patients have been in the ICU on ventilators for weeks and their prognosis for survival is poor, and now three new patients need those ICU beds and ventilators, including an ICU nurse and a respiratory therapist both of whom contracted COVID-19 working sacrificially in that same overwhelmed ICU? Any number of situations could be described which make it apparent that, while saving the most lives possible should definitely be a part of our decision-making, that consideration is insufficient by itself.

The "most life-years gained" strategy recognizes some of the concerns raised above. Saving five people who are expected to live for another 40 years each instead of saving five people who are expected to live 4 years each seems straightforward. But this approach also has its limitations. Considering only the most life-years gained does not fully account for the distribution of those life-years. For example, is giving twenty people one more year to live equal to giving four people another 5 years to live? Is giving one year to live to a 70-year-old equivalent to giving that one year to a 20-year-old? As with the most lives saved approach, the most life-years gained approach is ethically attractive and it should be a part of an allocation calculus, but it too is insufficient by itself.

\section{Treating people equally}

Treating people equally has moral appeal. Each person's desire to stay alive is given the same importance. Every person is granted the same moral status. There are two common 
approaches to applying equal treatment, the "lottery system," and "first-come, first-served."

The "lottery system" is quick and easy, it requires little knowledge of the individuals, and it resists corruption. However, it does not take into account relevant knowledge of the individuals, which should have an impact on resource allocation during a pandemic. For example, this approach does not consider a person's prognosis with and without treatment, a person's expected life-years, or whether an individual is critically needed to help save others. During a pandemic, allocation by simple lottery is insufficient by itself but it can be a component within a larger systems approach, especially when adjudicating resource allocation between individuals who have similar expected treatment benefit.

A second approach that endeavors to treat people equally is "first-come, first-served." Unfortunately, this approach has some flaws that are unique to disaster scenarios. Firstly, it does not actually treat all people equally, even though that is the intent. It favors more advantaged members of society, those who have access to information, those who can travel to areas with better resources, and those who can get to the limited resources first. In other words, it favors those with financial resources, influence, or connections. Secondly, it can ironically favor members of communities who are noncompliant with pandemic mitigation strategies like stay at home orders and quarantines and, as a result, these folks become infected sooner and get to the resources sooner. Finally, the "first-come, first-served" approach also does not maximize the benefits of available resources. For example, if a "first-served" patient consumes a substantial and ongoing amount of limited resources, then several patients who present later might be denied those same life-saving resources. Removing resources from one early patient with a poor prognosis to provide benefit to several subsequent patients with a better prognosis might be a very difficult, but ethically justifiable choice under the circumstances of a pandemic.

\section{Rewarding instrumental value}

Personnel can be one of the limited resources during a pandemic. Personnel, including but not limited to certain health care professionals, first responders, and essential scientists directly involved in diagnosis and treatment, can be of direct value to helping everyone in a community overcome the pandemic. Examples might include ICU physicians and nurses, respiratory therapists, infectious disease specialists, and the like. When these people fall ill to the pandemic, providing them medical care and getting them back into the workforce quickly benefits everyone. They have an "instrumental value" to society. Decision-making during pandemics should include provisions for providing treatment resources to those who are valuable to society in combating the pandemic.
It is worth pointing out that "social value" is different than instrumental value. Some individuals may be beneficial to the society as a whole, but they provide no direct benefit specific to fighting a pandemic. The social value of an individual is based upon other conventional and respected societal values. Social value alone should not direct the allocation of scarce resources during a disaster.

Also, during pandemics and disasters, there are workers who voluntarily and altruistically accept, or who are conscripted into, tasks that place them at an elevated personal risk, including risk of illness or death. These people should be assured by health care institutions that their instrumental value and their personal sacrifice will not go unnoticed if they fall ill and need care. This reciprocity is important to maintaining an engaged and committed work force during difficult times.

\section{Giving priority to the worst off}

Giving priority to the patients that are worst off is another algorithm, one that is applied daily during normal times. A patient who presents to the emergency department with acute chest pain on a normal day is moved to the front of the line as others with less critical needs wait. Another example is prioritizing individuals on organ transplant lists. Treating the worst off first in such situations usually needs little moral justification. However, a limited resource environment challenges this practice. For example, a patient might present to the ED in respiratory distress and undergo emergent intubation and placement on a ventilator, while several other patients with less acute respiratory symptoms wait. Continuing this otherwise standard practice during a pandemic would not take into account other relevant concerns, including the post-treatment prognosis of the patient who is worst off. If this person has a poor prognosis for recovery and liberation from the ventilator, the patient might require a significant amount of resources and prolonged mechanical ventilation for weeks, resources that might be in short supply during a pandemic. These resources would then be unavailable to others, several of whom might survive if those critical resources were redirected to them. Giving priority to the worst off may be morally appropriate and medically preferred in a well-supplied resource setting, but it may not provide good guidance in a limited resource setting, where maximizing the benefits from available resources is important.

In some situations, infants might be considered the worst off in that they have the most life-years to lose if they die prematurely. Directing resources first to the very young has appeal, but it is limited in that it also ignores prognosis. For some pandemics, influenza might be an example, if therapeutics are limited, directing those therapies to infants with a poor prognosis would offer less overall benefit than directing 
them to school age or adolescent patients with a better prognosis. This approach also categorically excludes the elderly. A modification of the youngest first approach considers maximizing the opportunity to live through the various "phases of life." For example, adolescents have already received a considerable societal investment of education and upbringing, and they have many life cycles ahead of them, phases of life that will not be experienced if cut short by a pandemic. Infants, on the other hand, neither have such an investment nor are they able to conceive of the life cycles that they will lose if their life is cut short. And, the elderly already have experienced most of their life cycles. So, infants and elderly would thus be of lower priority based on this approach. In short, considering infants as the worst off may be a component of a decision calculus, but it cannot always be the primary determinant of care.

\section{Treating patients with non-pandemic illnesses during a pandemic}

Finally, during a pandemic, people still need medical care for illnesses that are not associated with the pandemic, like accidents and trauma, heart attacks and strokes, cancer, and serious infections. Decisions about what care these patients receive and when they receive it must consider both the pandemic patients and the non-pandemic patients. Patients presenting with non-pandemic conditions should not be favored over those presenting from the pandemic, neither should they be less favored. This challenge is relevant to all health care providers who are providing front-line care.

\section{Surgical care and COVID-19}

Clinical and surgical practices which are standard in normal times may not be standard or safe during COVID-19. How can we best adapt as physicians and surgeons? A number of leading surgical associations have recently released documents that provide some early guidance (SAGES, EAES, American College of Surgeons, Royal College of Surgeons, and others). There are several common themes in these documents.

On March 30, 2020, SAGES/EAES provided "Recommendations Regarding Surgical Response to COVID-19 Crisis", which, in part, states: All elective surgical and endoscopic cases should be postponed at the current time. There are different levels of urgency related to patient needs, and judgment is required ... the surgical care of patients should be limited to those whose needs are imminently life threatening, with malignancy that could progress or with active symptoms that could require urgent care.... This minimizes risk to both patient and health care team, as well as minimizes utilization of necessary resources, such as beds, ventilators, PPE, and unexposed health care providers and patients.

On April 7, 2020, SAGES/EAES followed their recommendations with a standard operating procedure document titled "Closing the Back Door, Recommendations in the Fight against COVID-19", which, in part, states: (1) it is extremely important to ... avoid unsuspected transmission by asymptomatic COVID-19 positive patients; and (2) Patients scheduled to undergo any type of surgical intervention should be screened for COVID-19 symptoms within $24 \mathrm{~h}$ of the scheduled intervention. In areas with a high clinical burden of COVID-19 infection, it is recommended that when possible all inpatients and outpatients scheduled for a surgical intervention undergo COVID-19 PCR testing within $24-48 \mathrm{~h}$ of the planned procedure. It is the responsibility of the surgical attending to ensure that testing is completed.

On April 6, 2020, seven UK societies weighed in with "Updated General Surgery Guidance on COVID-19," which, in part, states the following: (1) COVID-19 should be sought in any patient referred acutely or needing emergency surgery: history, COVID-19 testing, and CXR can assist; (2) Any patient currently prioritized to undergo urgent planned surgery must have self-isolated and be assessed for COVID19 as above; and (3) Where non-operative management is possible and reasonable (such as for early appendicitis and acute cholecystitis) this should be implemented.

Though these practices during the COVID-19 crisis are altered compared to standard care, they are consistent with the ethical principles applied during pandemics. For example, redirecting hospital beds, ICU beds, and PPE away from elective surgical patients to pandemic patients is consistent with maximizing benefits of limited resources. Treating acute appendicitis and cholecystitis with antibiotics whenever possible rather than with surgery similarly may maximize benefits. Testing patients needing urgent surgery for SARS-CoV-2 by PCR within $48 \mathrm{~h}$ of surgery not only helps ensure patient safety, these measures also help health care professionals avoid being infected by SARS-CoV-2 (Instrumental Value). When we understand the rationale and ethical underpinning of these differences, we may find the pandemic-induced changes less stressful.

\section{Transparency, trust, and clinical triage teams}

Patients, families, and others in society also should understand that clinical and surgical practices which are standard in normal times may not be standard or safe during a pandemic. This can be very difficult for them. This is especially difficult for families whose loved ones are critically or terminally ill from COVID-19. Since visitors may not be allowed in the ICU during these circumstances, goals of care 
discussions can be wrenching for family members, intellectually and emotionally. Family members without medical training may not fully understand the limits of medicine when this illness becomes severe. Their trust in the medical community will be better maintained when they receive frequent, honest, accurate, and transparent communication, and when they see the medical community consistently applying the treatment and triage practices being professed. An informed and trusting public is a valuable partner to the medical community during a pandemic.

Finally, for the most difficult decisions which may arise during a pandemic or other disaster, a clinical triage team is an enormously valuable resource. Respecting the four fundamental ethical principles, and applying and balancing the values described above (maximizing benefits, instrumental value, etc.) into an allocation framework, giving proper weight to each, is not easy. There is no one fundamental value that can be applied to a pandemic that will perfectly determine the just distribution of limited resources or the most appropriate clinical decisions. It is the application of multiple values, each informative yet individually insufficient, that guides us. The weight given to each one of these values will vary from one health care emergency to another, from one location or community to another, and from one time point in the pandemic to another. This is why clinical treatment organizations (hospitals and clinics) should strongly consider establishing a locally designated triage team working with multiple stakeholders in developing an allocation framework to guide decisions. These teams can then help make the difficult decisions, relieving the frontline health care professionals of having to do so. During a pandemic or a disaster, health care professionals may be physically and emotionally exhausted, and they also may become very personally invested in the care of individual patients. It is not fair, appropriate, or operationally sound to ask them to make the hard decision of resource allocation on their specific patients. Relying on health care professionals in the trenches to make the decision to remove resources from a patient for whom they have cared and invested deeply will likely increase their own personal stress and grief. This decision-making responsibility should therefore be the responsibility of clinical triage teams. Implementing these teams should enhance objectivity in the decision-making process, reduce conflicts of interest, and decrease the moral distress of front-line providers.

\section{Conclusion}

Allocating limited resources and altering clinical practices during health care emergencies and pandemics may be unfamiliar to some physicians, surgeons, and health care professionals. The fundamental ethical principles in health care are beneficence, justice, autonomy, and non-maleficence. These are still applicable during a pandemic. But, their application shifts as health care transitions from having its focus in caring for individuals to caring for society as a whole. Maximizing benefits from available resources, treating people equally, recognizing instrumental value, and prioritizing the worst off are established values. Each of these values has strengths and limitations, and tradeoffs are necessary. Choosing how each one of these values will have their appropriate say in how to allocate limited resources is where ethical and clinical reasoning skills need to be at their finest. Clinical and surgical practices which are standard in normal times may not be standard or safe during pandemics. Several leading surgical associations have recently released documents that provide some early guidance for dealing with COVID-19. Though the surgical and clinical practices proposed are altered compared to standard care, they are consistent with the ethical principles and values applied during pandemic care. Health care professionals, patients and families, and others in society benefit by understanding the rationale and ethical support for these clinical and surgical practices. Patient and societal trust in the medical community is better maintained when they receive accurate, honest, and transparent communication and when they see the medical community consistently applying the treatment and triage practices being professed. And, for the most difficult decisions which may arise during a pandemic, a clinical triage team can serve an enormously valuable role.

Acknowledgements The authors would like to thank Aurora Pryor MD MBA, Fred Brody MD MBA, and John Mellinger MD for their critical review and helpful suggestions.

Author contributions AR and PS involved in conceptualization and drafting the manuscript. AR, LB, AF, HA, and PS participated in editing and final approval.

Funding No financial support was received for this work.

\section{Compliance with ethical standards}

Conflict of interest Horacio Asbun discloses consulting fees from Verb Surgical, honoraria from Olympus and Boston Scientific, a non-paid consulting role with Storz, and a non-paid Advisory Committee role with Sirtecs. Phillip Shadduck discloses consulting fees from Teleflex Medical (R\&D) and Heron Therapeutics (Research and Advisory). Arthur Rawlings, Lea Brandt and Alberto Ferreres have no conflicts of interest or financial ties to disclose.

Ethical approval IRB approval was not required.

Research involving human and animal rights This work did not involve human subject research or protected health information. 


\section{Further resources}

American College of Surgeons (2020) Ethical considerations: an ethical framework for the allocation of resources in the event of shortages. https://www.facs.org/covid19/newsletter/033120/ethic s\#framework. Accessed 6 Apr 2020

American Medical Association (2020) Allocating limited healthcare resources: code of medical ethics opinion 11.1.3. https://www. ama-assn.org/delivering-care/ethics/allocating-limited-healt h-care-resources. Accessed 6 Apr 2020

American Medical Association (2020) Physician's Response in Disaster Response \& Preparedness: code of Medical Ethics Opinion 8.3. https://www.ama-assn.org/delivering-care/ethics/physicians -responsibilities-disaster-response-preparedness. Accessed 6 Apr 2020

Emanuel EJ, Persad G, Upshur R et al (2020) Fair allocation of scarce medical resources in the time of Covid-19. N Engl J Med. https:// doi.org/10.1056/NEJMsb2005114

National Academies of Sciences, Engineering, and Medicine (2020) Rapid expert consultation on crisis standards of care for the covid19 pandemic (March 28 2020). The National Academies Press, Washington, DC

Persad G, Wertheimer A, Emanuel EJ (2009) Principles for allocation of scarce medical interventions. Lancet 373:423-431
Royal College of Surgeons of Edinburgh et al (2020) Updated General Surgery Guidance on COVID-19. https://www.rcsed.ac.uk/newspublic-affairs/news/2020/april/updated-general-surgery-guidanceon-covid-19-2nd-revision-7th-april-2020. Accessed 6 Apr 2020

SAGES (2020) SAGES recommendations regarding surgical response to COVID-19 Crisis. http://www.sages.org. Accessed 11 Apr 2020

SAGES/EAES (2020) SAGES/EAES “Closing the Back Door" recommendations in the fight against COVID-19. https://eaes.eu/sages -eaes-closing-the-back-door-recommendations-in-the-fight-again st-covid-19. Accessed 11 Apr 2020

Truog RD, Mitchell C, Daley GQ (2020) The toughest triage-allocating ventilators in a pandemic. $\mathrm{N}$ Engl J Med. https://doi. org/10.1056/NEJMp2005689

White DB, Katz MH, Luce JM et al (2009) Who should receive life support during a public health emergency? Using ethical principles to improve allocation decisions. Ann Intern Med 150(2):132-138

Wölfel R, Corman VM, Guggemos W et al (2020) Virological assessment of hospitalized patients with COVID-2019. Nature. https:// doi.org/10.1038/s41586-020-2196-x

Publisher's Note Springer Nature remains neutral with regard to jurisdictional claims in published maps and institutional affiliations. 